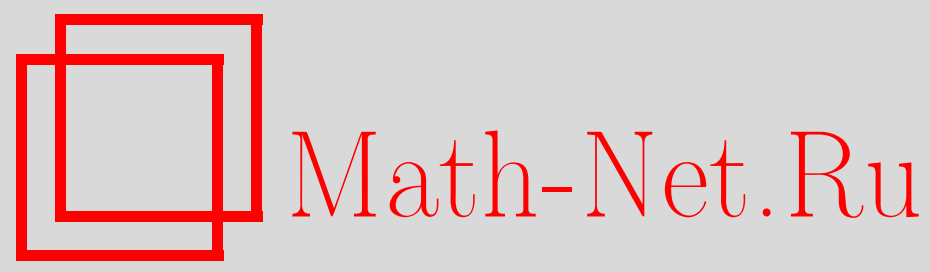

В. А. Клячин, Новые примеры трубчатых минимальных поверхностей произвольной коразмерности, Матем. заметкu, 1997, том 62, выпуск 1, 154-156

DOI: https://doi.org/10.4213/mzm1600

Использование Общероссийского математического портала Math-Net.Ru подразумевает, что вы прочитали и согласны с пользовательским соглашением http://www . mathnet.ru/rus/agreement

Параметры загрузки:

IP: 107.22 .136 .117

26 апреля 2023 г., 13:54:31

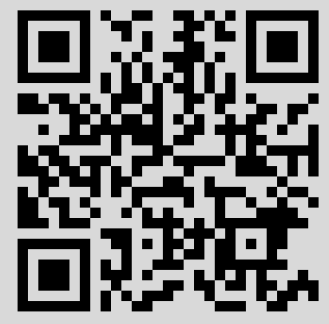




\section{НОВЫЕ ПРИМЕРЫ ТРУБЧАТЫХ МИНИМАЛЬНЫХ ПОВЕРХНОСТЕЙ ПРОИЗВОЛЬНОЙ КОРАЗМЕРНОСТИ}

\section{В. А. Клячин}

1. Пусть $M-p$-мерное связное некомпактное $C^{2}$-гладкое многообразие. Рассмотрим поверхность $\mathscr{M}=(M, u)$, заданную $C^{2}$-погружением $u: M \rightarrow \mathbb{R}^{n+1}, 2 \leqslant p \leqslant n$.

Поверхность $\mathscr{M}$ называется минимальной, если ее вектор средней кривизны $H$ тождественно равен нулю.

Пусть $M$ - многообразие без края. Поверхность $\mathscr{M}=(M, u)$ будем назьвать $т р у б$ чатой с проекцией $(\alpha, \beta)$ на направление, задаваемое вектором $е \in \mathbb{R}^{n+1}$, если множества

$$
\Sigma(t)=\{m \in M:\langle u(m), e\rangle=t\}, \quad t \in(\alpha, \beta),
$$

непусты, а всякая порция $u(M)$, заключенная между $\Sigma\left(t_{1}\right)$ и $\Sigma\left(t_{2}\right)$, предкомпактна.

Для произвольного $p \geqslant 2$ полагаем

$$
\Phi_{p}(t)=\int_{1}^{t}\left(y^{2(p-1)}-1\right)^{-1 / 2} d y, \quad t \geqslant 1 .
$$

Заметим, что $\varphi_{p}=\Phi_{p}(+\infty)<+\infty$ при всех $p \geqslant 3$.

Простейшими примерами трубчатых минимальных поверхностей служат гиперповерхности вращения в $\mathbb{R}^{n+1}$, задаваемые уравнением

$$
\lambda|\langle x, e\rangle|=\Phi_{p}\left(\lambda \sqrt{|x|^{2}-\langle x, e\rangle^{2}}\right),
$$

где $\lambda>0$-постоянная.

В работе [1] изучались свойства трубчатых минимальных гиперповерхностей. В частности, было доказано, что такие поверхности в случае $\operatorname{dim} \mathscr{M}>2$ расположены в слое между двумя параллельными гиперплоскостями, и дана точная оценка расстояния между ними. Поясним последнее. Компактность сечений $\Sigma(t)$ позволяет ввести на $(\alpha, \beta)$ функцию

$$
\rho(t)=\max _{m \in \Sigma(t)}\left(|u(m)|^{2}-t^{2}\right)^{1 / 2} .
$$

Обозначим через $\rho_{0}$ величину $\inf _{(\alpha, \beta)} \rho(t)$. B [1] доказана оценка

$$
\beta-\alpha \leqslant 2 \rho_{0} \varphi_{n}
$$

для трубчатых минимальных гиперповерхностей $\operatorname{dim} \mathscr{M} \geqslant 3$. Там же доказано, что если в (2) имеет место равенство, то $\mathscr{M}$ есть поверхность вращения вида (1) с $\lambda=1 / \rho_{0}$.

Случай поверхностей codim $\mathscr{M}>1$ рассматривался в [2], [3]. В [2] было доказано, что минимальные трубчатые поверхности размерности $p \geqslant 3$ также расположеныв слое между двумя параллельными гиперплоскостями, а в [3] дана точная оценка расстояниям

Работа выполнена при финансовой поддержке Российского фонда фундаментальных исследований, грант № 93-011-176. 
между ними. Другими словами, в [3] получено неравенство, аналогичное неравенству в [1]:

$$
\beta-\alpha \leqslant 2 \rho_{0} \varphi_{p}
$$

Однако, остался открытым вопрос: существуют ли трубчатые минимальные поверхности $\operatorname{codim} \mathscr{M}>1$, отличные от поверхностей вида (1), для которых в (3) имеет место равенство? Целью настоящей работы и является построение таких примеров.

Следует отметить также, что трубчатые минимальные поверхности с нетривиальными группами симметрий изучались в [4].

2. Пусть $\left\{e_{i}\right\}_{i=0}^{n}$ - ортонормированньй базис в $\mathbb{R}^{n+1}$, а $x_{0}, x_{1}, \ldots, x_{n}$ - соответствуюшие координаты. Через $S^{n-1}$ мы обозначим сферу радиуса 1

$$
S^{n-1}=\left\{x \in \mathbb{R}^{n+1}: x_{0}=0, x_{1}^{2}+\cdots+x_{n}^{2}=1\right\} .
$$

Рассмотрим $(p-1)$-мерную поверхность, лежащую на $S^{n-1}$ и заданную $C^{2}$-погружением $R: F \rightarrow S^{n-1} \subset \mathbb{R}^{n+1}$ компактного многообразия $F$.

Зафиксируем произвольньй интервал $(\alpha, \beta)$ и $C^{2}$-функцию $r(t)$, определенную на $(\alpha, \beta)$. Тогда можно построить $p$-мерную поверхность $\mathscr{M}$, заданную $C^{2}$-погружением $u: F \times(\alpha, \beta) \rightarrow \mathbb{R}^{n+1}$ таким образом, что если $y \in F, t \in(\alpha, \beta)$, то

$$
u(y, t)=r(t) R(y)+t e_{0} .
$$

Основной результат заметки составляет

ТЕОремА. Поверхность $\mathscr{M}$ вида (4) является минимальной трубкой с проекиией $(\alpha, \beta)$ в том и только том случае, когда $R: F \rightarrow S^{n-1}$ - минимальное погружение в сферу, а функиия $r(t)$ является решением дифференциального уравнения

$$
r^{\prime \prime}(t) r(t)=(p-1)\left(1+{r^{\prime}}^{2}(t)\right) .
$$

ДокАЗАТЕЛЬСтво. Прежде всего ясно, что для поверхностей вида $(4)$ сечения $\Sigma(t)$ лежат на $(n-1)$-мерньх сфферах $S(t)$ радиуса $r(t)$. Пусть $\nabla, D, \bar{\nabla}$ - связности на $\mathscr{M}$, сфере $S(t)$ и $\mathbb{R}^{n+1}$ соответственно, а $B, B^{\prime}$ и $b$ - вторые фундаментальные формы $\mathscr{M}$ в $\mathbb{R}^{n+1}$, сечения $\Sigma(t)$ в $S(t)$ и гиперповерхности $W$ вращения графика функции $r(t)$ вокруг оси $O x_{0}$. Выберем в нормальном пространстве к $\mathscr{M}$ базис $\xi_{0}, \xi_{1}, \ldots, \xi_{n-p}$ таким образом, что $\xi_{1}, \ldots, \xi_{n-p}$ - ортонормированньй базис в нормальном пространстве к сечению $\Sigma(t)$ в сфере $S(t)$, а $\xi_{0}$ - вектор внешней единичной нормали к $W$. В касательном пространстве к $\mathscr{M}$ базис выберем так, что $X_{1}, X_{2}, \ldots, X_{p-1}-$ касательные к $\Sigma(t)$, а $X_{0}$ направлен по меридиану гиперповерхности вращения $W$.

Тогда, используя определения вторых фундаментальных форм [5], получим для всех $k=1,2, \ldots, p-1$ и $i=1,2, \ldots, n-p$

$$
\begin{gathered}
\left\langle B\left(X_{k}, X_{k}\right), \xi_{i}\right\rangle=\left\langle\bar{\nabla}_{X_{k}} X_{k}, \xi_{i}\right\rangle=\left\langle D_{X_{k}} X_{k}, \xi_{i}\right\rangle=\left\langle B^{\prime}\left(X_{k}, X_{k}\right), \xi_{i}\right\rangle \\
\left\langle B\left(X_{0}, X_{0}\right), \xi_{i}\right\rangle=\left\langle\bar{\nabla}_{X_{0}} X_{0}, \xi_{i}\right\rangle=0
\end{gathered}
$$

а для всех $k=0,1, \ldots, p-1$

$$
\left\langle B\left(X_{k}, X_{k}\right), \xi_{0}\right\rangle=\left\langle\bar{\nabla}_{X_{k}} X_{k}, \xi_{0}\right\rangle=\left\langle b\left(X_{k}, X_{k}\right), \xi_{0}\right\rangle
$$


Заметим, что $\left\{X_{k}\right\}$ - главные направления поверхности $W$, и если $\left\{\lambda_{k}\right\}$ - соответствующие главные кривизны, то

$$
\begin{aligned}
\left\langle b\left(X_{0}, X_{0}\right), \xi_{0}\right\rangle & =\lambda_{0}=\frac{r^{\prime \prime}(t)}{\left(1+r^{\prime 2}(t)\right)^{3 / 2}}, \\
\left\langle b\left(X_{k}, X_{k}\right), \xi_{0}\right\rangle & =\lambda_{k}=-\frac{1}{r(t)\left(1+r^{\prime 2}(t)\right)^{1 / 2}}, \quad k=1,2, \ldots, p-1 .
\end{aligned}
$$

Теперь если обозначить через $\mathscr{H}(t)$ вектор средней кривизны сечения $\Sigma(t)$ в сфере $S(t)$, то

$$
\left\langle H, \xi_{i}\right\rangle=\left\langle\mathscr{H}(t), \xi_{i}\right\rangle, \quad\left\langle H, \xi_{0}\right\rangle=\frac{r^{\prime \prime}(t)}{\left(1+{r^{\prime}}^{2}(t)\right)^{3 / 2}}-\frac{p-1}{r(t)\left(1+r^{\prime 2}(t)\right)^{1 / 2}} .
$$

Откуда следует, что (5) и минимальность погружения $R: F \rightarrow S^{n-1}$ эквивалентна минимальности поверхности $\mathscr{M}$.

Определим на интервале $\left(-\varphi_{p}, \varphi_{p}\right)$ функцию $\Psi_{p}(t)$ следующим образом. На $\left[0, \varphi_{p}\right)$ рассмотрим функцию, обратную к $\Phi_{p}(t)$, а $\Psi_{p}(t)$ построим как четное продолжение последней на интервал $\left(-\varphi_{p}, \varphi_{p}\right)$. Прямыми вычислениями проверяется, что $\Psi_{p}(t)$ удовлетворяет уравнению (5). Поскольку inf $\left(-\varphi_{p}, \varphi_{p}\right) \Psi_{p}(t)=\Psi_{p}(0)=1$, то нетрудно видеть, что поверхности вида $(4)$ с $r(t)=\Psi_{p}(t)$ и произвольным компактным подмногообразием $R: F \rightarrow S^{n-1}$ дают необходимые примеры.

ЗАмЕчАниЕ. Примеры нетривиальных замкнутьх минимальных подмногообразий сферы $S^{n-1}$ можно найти в работах [6]-[8]. Один из таких примеров получается в пересечении сферы $S^{n-1}$ и конуса Саймонза, задаваемого уравнением

$$
x_{1}^{2}+x_{2}^{2}+x_{3}^{2}+x_{4}^{2}=x_{5}^{2}+x_{6}^{2}+x_{7}^{2}+x_{8}^{2} .
$$

\section{СПИСОК ЦИТИРОВАННОЙ ЛИТЕРАТУРЫ}

1. Веденяпин А. Д., Миклюков В. М. // Матем. сб. 1986. Т. 131. № 2. С. 240-250. 2. Миклюков В. М., Ткачёв В. Г. // Матем. сб. 1989. Т. 180. №9. С. 1278-1295. 3. Клячин В. А. // Сиб. матем. ж. 1992. Т. 33. № 4. С. 201-205. 4. Фоменко А. Т. Вариационные методы в топологии. М.: Наука, 1982. 5. Кобаяси Ш., Номидзу К. Основы дифференциальной геометрии. T. 2. M.: Наука, 1981. 6. Simons J. // Ann. of Math. 1968. V. 88. № 2. P. 62-105. 7. Gauchman H. // Trans. Amer. Math. Soc. 1986. V. 298. № 2. P. 779-791. 8. Chern S.-S., do Carmo M., Kobayashi S. // Functional Analysis and Related Fields. Berlin-New York: Springer Verlag, 1970. P. 59-75. 